\title{
Attenuation Tomography of the Yellow Sea/Korean Peninsula from Coda-source normalized and direct Lg Amplitudes
}

\author{
Sean R. Ford, ${ }^{1}$ William S. Phillips, ${ }^{2}$ William R. Walter, ${ }^{1}$ Michael E. Pasyanos, ${ }^{1}$ Kevin Mayeda, ${ }^{3}$ \\ and Douglas S. DREgER ${ }^{4}$
}

\begin{abstract}
We invert for regional attenuation of the crustal phase Lg in the Yellow Sea/Korean Peninsula (YSKP) using three different amplitude attenuation tomography methods. The first method solves for source, site, and path attenuation. The second method uses a scaling relationship to set the initial source amplitude and interpret the source term after inversion. The third method implements a coda-derived source spectral correction. By comparing methods with slightly different assumptions we are able to make a more realistic assessment of the uncertainties in the resulting attenuation maps than is obtainable through formal error analysis alone. We compare the site, source and path-terms produced by each method and comment on attenuation, which correlates well with tectonic and topographic features in the region. Source terms correlate well with each other and with magnitude. Site terms are similar except for two stations that are located in a region that has the greatest difference in path term, which demonstrates the site/path trade-off. Another region of path term difference has the fewest crossing paths, where the tomography method employing the coda-derived spectral correction may perform more accurately since it is not as susceptible to the source/ path trade-off. The Bohai Bay basin, an area of extension, is a region of high attenuation, and regions of low attenuation occur along topographic highs located in the Da-xin-an-ling and Changbai Mountains and Mount Taishan.
\end{abstract}

\section{Introduction}

There are many reasons to study the attenuation of seismic waves. Variations in regional attenuation $(1 / Q)$ can facilitate structure and tectonic interpretation (e.g., Frankel, 1990). Local and regional

\footnotetext{
${ }^{1}$ Lawrence Livermore National Laboratory, Livermore, CA 94550, USA. E-mail: sean@1lnl.gov

${ }^{2}$ Los Alamos National Laboratory, Los Alamos, NM 87545, USA.

${ }^{3}$ Weston Geophysical, Lexington, MA 02420, USA.

${ }^{4}$ Berkeley Seismological Laboratory, Berkeley, CA 94720, USA.
}

distance attenuation of seismic phases is important in earthquake hazard prediction. Quantifying seismic wave attenuation and correcting for its effects improves source parameter studies, which will aid in discrimination of small nuclear tests from naturally occurring earthquakes (e.g., BAKER et al., 2004; MAyeda et al., 2003; TAYLOR et al., 2002). Current event identification relies on $Q$ models to remove propagation effects, revealing source differences. $Q$ models taken from the literature can vary greatly within the same region. In previous work (FORD et al., 2008), we compared five different 1-D methods to measure $Q_{\mathrm{Lg}}$ and attempted to assess the error associated with the results. The assessment showed the possible influence of lateral variations in attenuation. In the same spirit of the 1-D method comparison study, we compare inversions for 2-D attenuation in the Yellow Sea/Korean Peninsula (YSKP) using three different methods with identical data. The comparison is made for the source, site, and path parameters.

Comparison of solutions obtained with different methods can give insight to the model error, which is often considerably more important and larger in magnitude than any type of random error that is usually calculated for inverse studies. This study will only assess this model-based error. In the section that follows we will outline the amplitude tomography method of Phillips and STEAD (2008) and the source-interpreted amplitude tomography method of PAsYanos et al. (2009). We will also introduce the coda-source corrected amplitude tomography method, which is a 2-D implementation of the 1-D analysis presented in WALTER et al. 
(2007). Concludingly, the attenuation structure of the YSKP will be interpreted in terms of the tectonics of the region.

\section{Data Set and Attenuation Tomography Techniques}

The YSKP data set consists of 145 earthquakes recorded at 6 broadband (20 sps) three-component stations of the Global Seismographic Network (GSN) and the OHP-Japan network (station TJN, Fig. 1). We omitted data with paths that traverse the Sea of Japan/East Sea, since this region is oceanic crust and does not efficiently propagate Lg (ZHANG and LAY, 1995). Using these data, we apply three different attenuation tomography techniques. By testing techniques that have different assumptions in the source, site and attenuation trade-offs we hope to better understand true uncertainties in the resulting $Q$ maps.

The first method we implement is the amplitude tomography method of PHILLIPS and STEAD (2008), which assumes that the spreading-corrected $\mathrm{Lg}$ spectrum $\left(A_{\mathrm{Lg}}\right)$ at a finite frequency $f$ can be represented as

$$
\begin{aligned}
\ln \left(A_{\mathrm{Lg}}\right)-\ln (G(r))= & \ln (S(f))+\ln (P(f)) \\
& -\frac{\pi f}{U} \int_{s} Q^{-1} d s,
\end{aligned}
$$

where $U$ is the group velocity, and is assumed to be $3.5 \mathrm{~km} / \mathrm{s}$. The inversion solves for $S(f)$ and $P(f)$, the source and site terms, respectively, as well as $Q^{-1}$ along the path, $s$, in a damped least-squares sense using the LSQR algorithm (PAIGE and SAUnders, 1982). Damping was chosen on the basis of L-curve analysis (HANSEN, 1998), in which the final damping parameter minimizes both the model length and error. The mean of the log site terms is damped to zero and the spreading correction is done using the STREET et al. (1975) function

$$
G(r)=\begin{array}{cc}
r^{-1} & \text { for } r<r_{0} \\
\frac{1}{r_{0}}\left(\frac{r_{0}}{r}\right)^{\gamma} & \text { for } r \geq r_{0}
\end{array},
$$

where $\gamma$ is 0.5 and $r_{0}$ is $100 \mathrm{~km}$. Therefore, spreading transitions from a spherical to a cylindrical type at approximately $100 \mathrm{~km}$. FORD et al. (2008) found that results differed only slightly when $r_{0}$ is between 60 and $120 \mathrm{~km}$. For brevity we will refer to this method as AMP (for AMPlitude).

We also implement the method of PAsyanos et al. (2009), which is similar in form to the AMP method [eq (1)], but which uses a starting source term based on seismic moment as defined in the magnitude distance amplitude correction (MDAC) formalism (WALTER and TAYLOR, 2001). The formalism employs a modified corner-frequency source model with frequency-squared fall-off incorporating seismic moment, apparent stress, and source-region geophysical parameters. In this way, the output source terms are perturbations to the original source, and the source term has a better physical interpretation. The site terms are not constrained. This method will be referred to as source interpretation (SI).

We also employ a recently developed method (WALTER et al., 2007), which alters the AMP method so that the amplitude is directly corrected for the source using stable, coda-derived source spectra that already account for regional source-scaling and are calculated via the methodology of MAYEDA et al. (2003). As performed in WALTER et al. (2007) on Italian data, the coda-derived source spectra have been calibrated specifically for the YSKP region and include non-self-similar source scaling determined independently using the coda ratio method which was first introduced by MAYEDA et al. (2007). Also, as with the SI method, the site terms are not constrained. This method will be referred to as coda source (CS).

Amplitude inversion methods based on eq. (1) must confront the direct trade-off of the site and source terms. The AMP method rebalances the terms by constraining the mean of the site terms to be zero, whereas the SI method rebalances by using a physically based initial source term. Other differences between the methods are that the AMP and CS methods use a first-difference roughening matrix in their regularization whereas the SI method uses second-difference regularization (finite-difference approximation of the Laplacian operator). The SI method uses a block discretization as opposed to the node-based discretization of the AMP and CS methods. A grid spacing of $1^{\circ}$ (blocks or nodes) was used for all methods due to the relatively small data set and to facilitate comparison. The choice of 

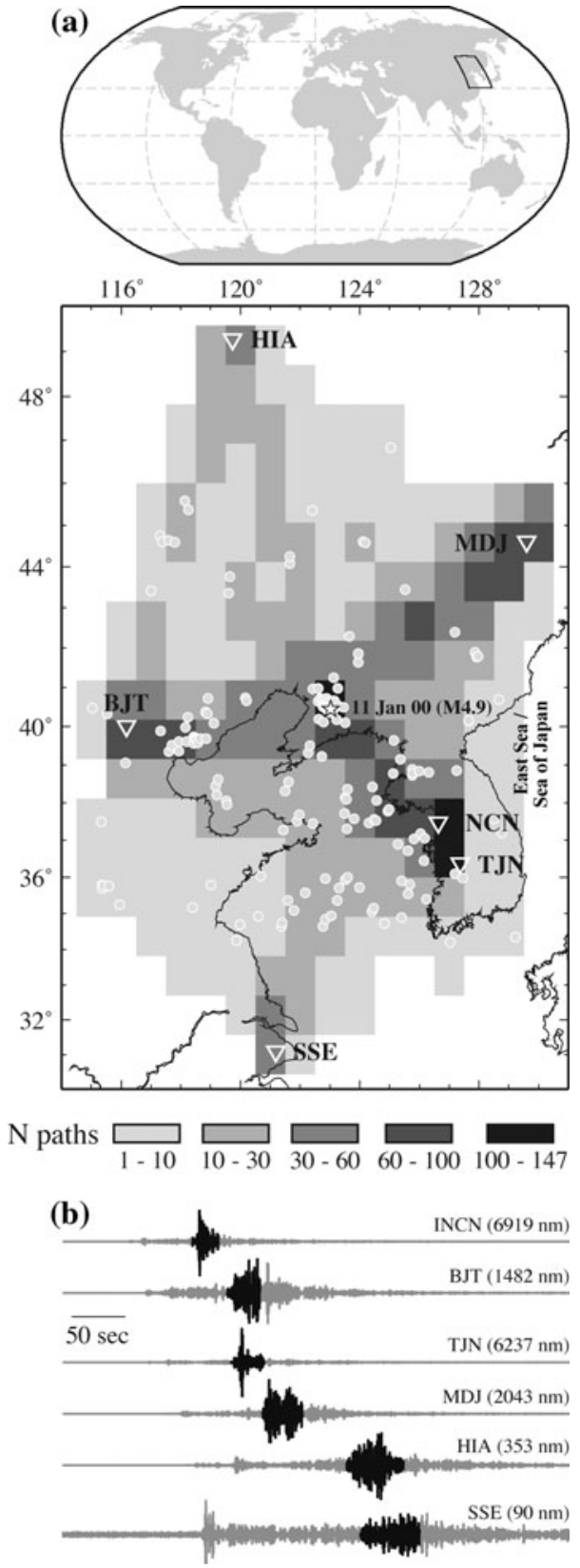

Figure 1

a YSKP region (outlined in global map) with events (circles), stations (inverted triangles), and path density (grayscale) for the data used in this study. b Example waveforms filtered in the band of interest (1-2 Hz) for a M4.9 event on $11 \mathrm{Jan} 00$ (star in a) to all stations. The $\mathrm{Lg}$ window is darkened relative to the rest of the waveform and the station name and maximum displacement are given at the end of each waveform

discretization will have little effect on the solution, though the regularization may make a difference and this will be investigated.
Data collection starts with an analyst review of each seismogram. The beginning of the $\mathrm{Lg}$ window is defined by the analyst pick, or when a pick is not available, the group velocity $3.45 \mathrm{~km} / \mathrm{s}$. The end of the window is defined by the group velocity $2.8 \mathrm{~km} / \mathrm{s}$, and the minimum window length is $1 \mathrm{~s}$. These windows are used to measure time-domain root-mean-squares amplitudes, which are converted to pseudo-spectral amplitudes in the passband of $1-2 \mathrm{~Hz}$ via the method of TAYLOR et al. (2002). Amplitudes are kept if the pre-event signal-to-noise ratio (SNR) exceeds two. A pre-phase SNR test resulted in only a few less amplitude measurements and was not used.

\section{Results and Discussion}

In the remaining sections attenuation will be discussed in terms of $q$ which is defined as $1000 / Q$, and is linearly related to attenuation so that high $q$ means high attenuation and low $q$ means low attenuation. $q$ values will also be translated to $Q$ to facilitate comparison with other studies. $q$ from the attenuation term of the new CS method is shown in Fig. 2. Attenuation derived with all methods is an apparent path attenuation, which will have components of both scattering and intrinsic attenuation, and we make no attempt to separate the two. Figure 2 shows attenuation that is correlated with topography (low $q$ in the Da-xin-an-ling and Changbai Mts., and Mt. Taishan) and basins (high $q$ in the Bohai Bay and Songliao Basin), where there is a transitional region along the Yellow Sea/West Sea from high $q$ in the west, a region of extension, to low $q$ in the east along the Korean coast. The $q$ in the entire YSKP ranges from $0.95(Q=1048)$ to $3.63(Q=275)$. Resolution of the path term is calculated via direct solution of the normal equations using Cholesky decomposition, and the resolution length is estimated by taking the square-root of the ratio of grid area to diagonal resolution element (Phillips and STEAD, 2008). This length is contoured in Fig. 2 and is approximately $3^{\circ}$ over most of the YSKP, and where there are no crossing rays, no $q$ is plotted.

The source terms output from the inversions is compared among each other and with $\mathrm{M}_{\mathrm{W}}$ in Fig. 3 (top right, panels $\mathrm{A}, \mathrm{B}, \mathrm{C}, \mathrm{E}, \mathrm{F}, \mathrm{I}$ ), where $\mathrm{M}_{\mathrm{W}}$ is either 


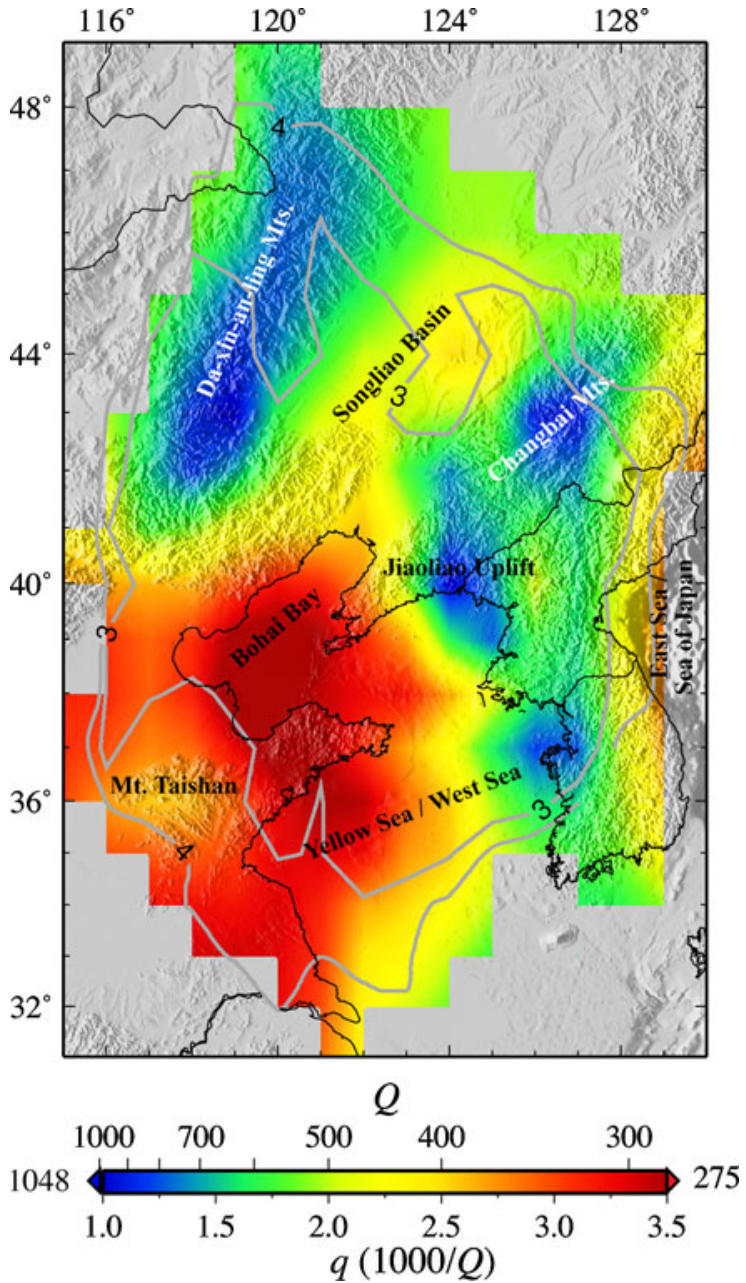

Figure 2

Coda-source corrected amplitude tomography of the YSKP region plotted on grayscale topographic gradient. The color scale is linear in $q(1000 / Q)$ with the minimum (275) and maximum (1048) $Q$ shown. Resolution contours of $3^{\circ}$ and $4^{\circ}$ are also plotted and only regions with crossing raypaths are imaged. Regional features are annotated and discussed in the text

a coda-based magnitude or has been derived from a source inversion (e.g., Herrmann et al., 2007). All methods correlate well with $\mathrm{M}_{\mathrm{W}}$ (top row, panels AC, Fig. 3). The CS method does not invert for the source, so the values given in Fig. 3 are the source terms used to correct the data for this method, thus as expected, these source terms agree best with catalog $\mathrm{M}_{\mathrm{W}}$. There is an outlier common to all methods that is circled in Fig. 3. This event has a catalog magnitude of 4.9, although a recent source inversion of this event calculated an $\mathrm{M}_{\mathrm{W}}$ of 4.7 (Herrmann et al.,
2007). Therefore, this outlier may be due to an incorrect catalog magnitude, which demonstrate that the SI method is able to correct for small errors in the initial source term. Also, as expected, the source terms of the AMP and SI method are very similar (panel F, Fig. 3) except for two outliers that are marked with a diamond and square. These two events are very near one another and located just south of station TJN (Fig. 1), which is a region of very low attenuation (see Fig. 2). The outlier marked with a square is an outlier for all AMP comparisons, and is the smallest event in the data set.

The site terms are mutually compared and with Vs30 in Fig. 3 (bottom left, panels D, G, H, J, K, L), where Vs30 for each station is taken from the topography-derived database of WALD and AlLEN (2009). We compare with Vs30 because it is often used as a proxy for site response in engineering applications and it would be useful to see if there is any correlation with Lg-derived site terms. There is very little correlation between the site term from the different methods and Vs30 (first column, panels D, G, J, Fig. 3), though there is a slight positive correlation with the SI method. The site terms of the CS and AMP method correlate fairly well (panel $\mathrm{H}$, Fig. 3), except for an absolute shift that is due to the constraint of the AMP method that requires the mean of the site terms to be zero. The site terms of the SI method agree well with the other methods (bottom row, panels J, K, L, Fig. 3), except for the site terms due to INCN and TJN. These stations are relatively close to one another and in a region of very low attenuation (see Fig. 2). However, the variation in these site terms is small and general agreement among the methods is good.

The path terms are compared amongst each other in Fig. 4. The panels along the diagonal of Fig. 4 (panels A, E, I) are the 2-D path terms for each method. The image produced with the CS method (panel E, Fig. 4) is the same as in Fig. 2 without bilinear interpolation of the values. Panels in the top right of Fig. 4 (panels B, C, F) compare $q$ values from each method. Each point in those panels represents $q$ at a specific grid point, or latitude/longitude point on the map. The location of grid point correlation can be found by looking at the bottom left of Fig. 4 (panels $\mathrm{D}, \mathrm{G}, \mathrm{H})$. These panels are 2-D plots of percent 


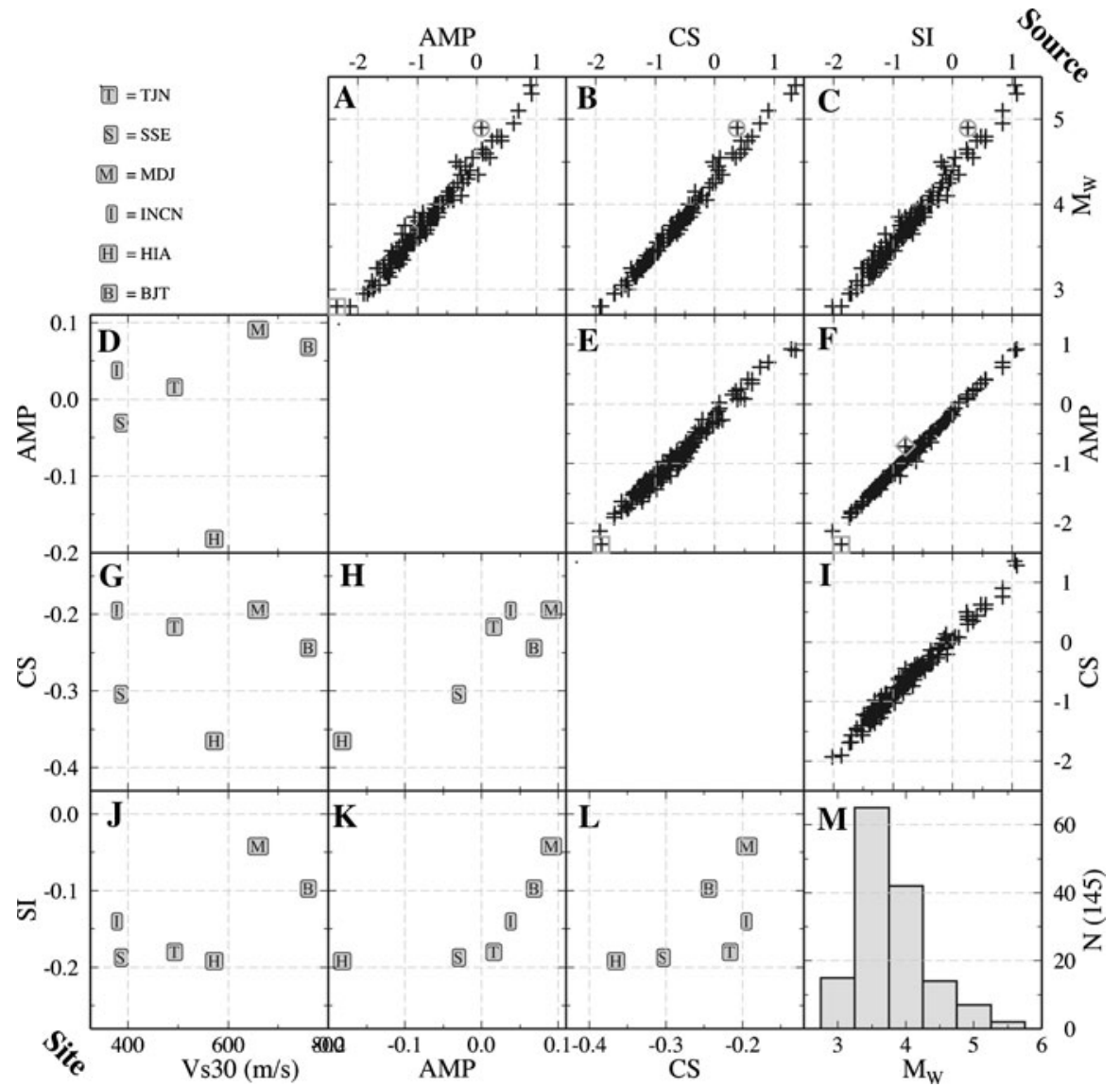

Figure 3

Source and site term comparison of the amplitude tomography method (AMP), coda-source corrected method $(C S)$ and source-interpreted method ( $S I$, see text for descriptions of the methods). Source comparison is shown in the top right panels (panels $A, B, C, E, F, I)$ where $\mathrm{M}_{\mathrm{W}}$ is from either a coda-source measurement or source mechanism inversion and a histogram of their values is in the bottom right corner (panel $M$ ). Outliers are marked and discussed in text. All panels share the same range. Site comparison is shown in the bottom left panels (panels D, $G, H$, $J, K, L)$ where Vs30 are values from WALD and ALLEN (2009) and points are given by station names (legend in top left corner). The absolute range of the panels is the same, though the minimum and maximum may vary slightly to facilitate comparison. Values are in log units (unless otherwise noted), and the SI and CS event terms are in log amplitude of the source spectra

difference $P D$ between two $q$ maps, $A$ and $B$. To make these plots we calculate

$$
P D_{i j}=\frac{2\left|A_{i j}-B_{i j}\right|}{A_{i j}+B_{i j}} \times 100,
$$

which is the absolute difference between two points divided by their average. The grid point by grid point $q$ of all the methods correlates well, especially between the CS and AMP methods (panel B). However, there is high $P D$ along the Da-xin-an-ling Mts. (panel D and see Fig. 2 for location), which is a region with few crossing paths (Fig. 1), Therefore the CS method may be better at resolving structure that is poorly sampled. This performance difference may be due to a reduction of the null space gained in the assumed elimination of the source term as a model parameter (Menke et al., 2006). The comparison with the SI method has more scatter (panels C, F) and this difference occurs along the northwest border of the tomography and in the region near stations INCN and TJN (highest $P D$ in panels $\mathrm{G}, \mathrm{H}$ ), where the SI method produces the smallest $q$ (panel I). The difference along the northwest border is due to the effects of the methods' different damping and regularization in an unconstrained region. The SI method does not deviate from the starting $q$ of the initial 


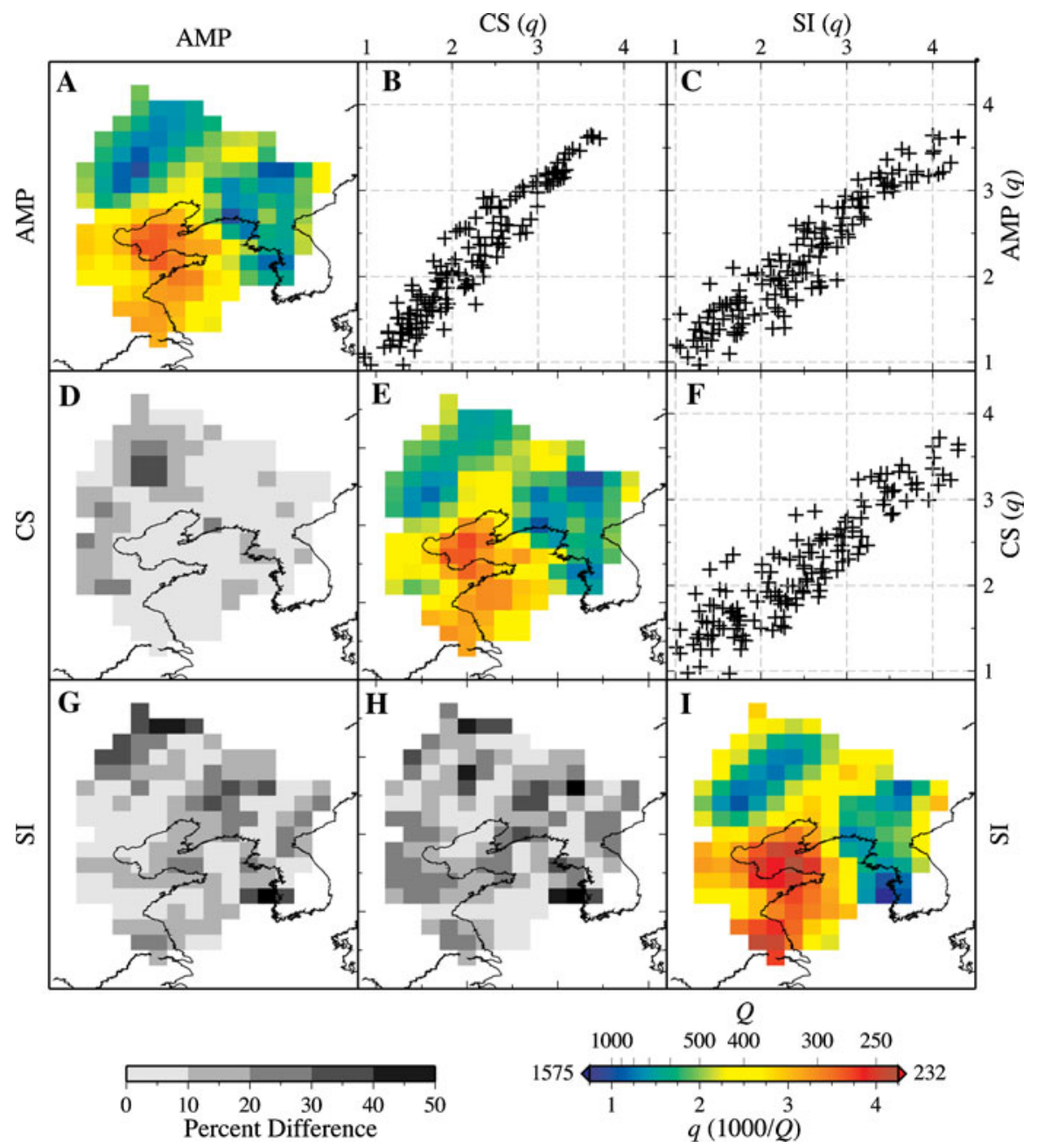

Figure 4

Path term comparison of the amplitude tomography method $(A M P)$, coda-source corrected method $(C S)$ and source-interpreted method (SI, see text for descriptions of the methods). Plots along the diagonal (panels $A, E, I$ ) show spatial attenuation for the YSKP region ( $q$ color scale in the lower right) for each method. Comparison at each $1^{\circ}$ grid node is shown in the top right panels (panels $B, C, F$ ) where values are given in $q(1000 / Q)$. Spatial comparison in normalized percent difference (scale in lower left) is shown in the bottom left panels (panels D, G, H)

model, and tightly constrains the low $q$ region to be along the Da-xin-an-ling Mts. The AMP method smooths the low $q$ of the Da-xin-an-ling Mountains to the unconstrained region, however this area is unresolved and therefore should not be interpreted. The difference in the region near stations INCN and TJN is most probably related to the source and site outliers discussed previously, and demonstrates the trade-off between source, site, and path inherent to the underlying formalism used by all methods. This is especially interesting when comparing the AMP and SI methods (panel G), which differ not only in how they resolve the source-site tradeoff but also in the regularization schemes employed. These differences remained even after perturbing the damping parameter around the optimum value to make the methods as similar as possible.

There are several previous lateral attenuation studies in which the YSKP region is imaged. PHILLIPS et al. (2005) produced maps of $\operatorname{Lg} Q$ at $1 \mathrm{~Hz}$ in Asia and where there is overlap with this study there is good agreement in spatial variation as well as absolute $q$. PEI et al. (2006) used amplitudes recorded to measure $\mathrm{M}_{\mathrm{L}}$ in southern China and inverted for $Q$ 
near $1 \mathrm{~Hz}$ in the region. The Bohai Bay is one of the most attenuating regions in their study, and this feature along with the low attenuation near Jiaoliao is similar to the results here. The $q$ near the Da-xin-anling and Changbai Mountains in this study is not as low as in PEr et al. (2006), though these features are at the edges of their model. In an update of an earlier study, Mitchell et al. (2008) calculate $\operatorname{Lg}$ coda $Q$ and its power-law frequency dependence for all of Eurasia. Though the YSKP region contains some of the greatest standard error in their study, they find high attenuation along the western portion of the Yellow Sea and Bohai Bay that is similar to this study. However, Mitchell et al. (2008) did not find the low $q$ features along the mountainous regions shown in Fig. 2. XIE et al. (2006) derive an $\operatorname{Lg} Q$ map of Eurasia using a two-station spectral ratio method. As with Mitchell et al. (2008), Xie et al. (2006) find agreement with most of the features in this study, but do not find a high $Q$ region along the Changbai Mountains, though there are few crossing paths in this region. Finally, Chung et al. (2007) spatially smoothed the results from a reverse two-station analysis of $\operatorname{Lg} Q$ to produce an image of $Q$ at $1 \mathrm{~Hz}$ for the YSKP. Their results differ from those presented here in the Songliao Basin and Bohai Bay, where they find low $q(<1.5)$. Hu et al. (2001) found high heat flow anomalies and evidence for a pull-apart basin in the Bohai Bay region which would predict high $q$ in this area relative to the surrounding region.

\section{Conclusion}

We introduce CS corrected amplitude tomography and compare it with two other similar methods to measure path attenuation in the Yellow Sea/Korean Peninsula region. The CS method is a 2-D implementation of the 1-D method of WALTER et al. (2007) and it compares favorably with the amplitude tomography method (AMP) of PhILLIPS and STEAD (2008) and a new SI amplitude tomography method developed by PAsyanos et al. (2009). The CS method corrects for the source using independent, stable coda-derived source spectra. The source term is estimated in the AMP and SI methods and additional steps are taken to balance the inherent source-site trade-off. The AMP method requires that mean of the site terms is zero, and the SI method uses a scaling relationship to set the initial source amplitude and interpret the source term after inversion. By comparing methods with different assumptions we are able to make a more realistic assessment of the uncertainties in the resulting attenuation maps than is obtainable through formal error analysis alone.

The maps of 2-D attenuation are fairly similar, indicating the results are robust to differences in regularization, damping, and source and site constraints. The methods also have similar reduction of variance. Due to its reduction of the null space from the elimination of the source parameter in the inversion, the CS method may be more accurate in regions with poor coverage. The source correction potentially improves coverage by adding events measured at only one station. Also, all methods are insensitive to small errors in the starting model. This is especially encouraging in the context of the SI method, where by amplitudes from new events can now be better predicted.

The greatest difference in the model parameters produced by each of the methods is due to the region between stations INCN and TJN in South Korea. The source, site, and path terms from this area have a slight variance among the methods. A higher resolution, more regional study is needed to find appropriate parameters for this subregion. Attenuation in the Yellow Sea/Korean Peninsula is correlated with topography (low attenuation) and extension in the Bohai basin (high attenuation). The $q$ in the entire YSKP ranges from $0.95(Q=1048)$ to 3.63 $(Q=275)$.

\section{Acknowledgments}

Data were processed with RBAP (Regional Bodywave Amplitude Processor) software developed at Lawrence Livermore National Laboratory (WALTER et al., 2004; RuPPERT et al., 2008). We thank the RBAP development team, D. Dodge, M. Gansberger, and E. Matzel. This research was performed under the auspices of the U.S. Department of Energy by the University of California, Lawrence Livermore National Laboratory (LLNL) under National Nuclear 
Security Administration contract W-7405-ENG-48. This is LLNL contribution LLNL-JRNL-411151 and BSL contribution 09-04.

Open Access This article is distributed under the terms of the Creative Commons Attribution Noncommercial License which permits any noncommercial use, distribution, and reproduction in any medium, provided the original author(s) and source are credited.

\section{REFERENCES}

BaKer, G. E., Stevens, J., and Xu, H. M. (2004), Lg group velocity: A depth discriminant revisited, Bull. Seismol. Soc. Am. 94, 722739.

Chung, T. W., Noh, M. H., Кiм, J. K., Рark, Y. K., Yoo, H. J., and LEES, J. M. (2007), A study of the regional variation of lowfrequency $Q_{L g}^{-1}$ around the Korean Peninsula, Bull. Seismol. Soc. Am. 97, 2190-2197.

Ford, S. R., Dreger, D. S., Mayeda, K., Walter, W. R., Malagnini, L., and Phillips, W. S. (2008), Regional attenuation in northern California: A comparison of five 1-D $Q$ methods, Bull. Seismol. Soc. Am. 98 (4), 2033-2046, doi:10.1785/ 0120070218.

FRANKEL, A. (1990), Attenuation of high-frequency shear waves in the crust: Measurements from New York State, South Africa, and Southern California, J. Geophys. Res. 95, 17441-17457.

Hansen, P.C. (1998), Rank-deficient and discrete ill-posed problems: Numerical aspects of linear inversion SIAM Monograph, Philadelphia.

Herrmann, R.B., Walter, W.R., and Pasyanos, M.E. (2007), Seismic source and path calibration in the Korean Peninsula, Yellow Sea and Northeast China, 29th Monitor. Res- Rev.: Ground-Based Nuclear Explosion Monitoring Technologies, Denver, Colorado.

Hu, S., O'Sullivan, P. B., Raza, A., and Kohn, B. P. (2001), Thermal history and tectonic subsidence of the Bohai Basin, northern China: a Cenozoic rifted and local pull-apart basin, Phys. Earth Planet Int 126 (3-4), 221-235, doi:10.1016/ S0031-9201(01)00257-6.

Mayeda, K., Hofstetter, A., O’Boyle, J. L., and Walter, W. R. (2003), Stable and transportable regional magnitudes based on coda-derived moment-rate spectra, Bull. Seis.mol. Soc. Am 93, 224-239.

Mayeda, K., Malagnini, L., and Walter, W.R. (2007), A new spectral ratio method using narrow band coda envelopes: Evidence for non-self-similarity in the Hector Mine sequence, Geophys. Res. Lett., doi:10.1029/2007GL030041.

Menke, W., Holmes, R. C., and XIE, J. (2006), On the nonuniqueness of the coupled origin time-velocity tomography problem, Bull. Seis. Mol. Soc. Am. 96 (3), 1131-1139, doi: 10.1785/0120050192.

Mitchell, B. J., Cong, L., and Exström, G. (2008), A continentwide map of $1-\mathrm{Hz}$ Lg coda $Q$ variation across Eurasia and its relation to lithospheric evolution, J. Geophys. Res. 113, B04303, doi:10.1029/2007JB005065.

Paige, C. C., and SAunders, M. A. (1982), Algorithm 583, LSQR: Sparse linear equations and least-squares problems, Trans. Math Software 8, 195-209.

Pasyanos, M. E., Matzel, E. M., Walter, W. R., and Rodgers, A. $\mathrm{J}$ (2009), Broadband Lg attenuation modeling in the Middle East, Geophys. J. Int. 177, 1166-1176, doi:10.1111/j.1365-246X.2009. 04128.x.

Pei, S., Zhao, J., Rowe, C. A., Wang S., Hearn, T. M., Xu, Z., Liu, H., and Sun, Y. (2006), $M_{L}$ amplitude tomography in North China, Bull. Seismol. Soc. Amer. 96, 1560-1566.

Phillips, W. S., Hartse, H. E. and Rutledge, J. T. (2005), Amplitude ratio tomography for regional phase $Q$, Geophys. Res. Lett. 32, L21301.

Phillips, W. S., and Stead, R. J. (2008), Attenuation of Lg in the western US using the USArray, Geophys. Res. Lett. 35, L07307, doi:10.1029/2007GL032926.

Ruppert, S., Dodge, D., Ganzberger, M., Hauk, M., and Matzel, E. (2008), Enhancing seismic calibration research through software automation and scientific information management, Proc 30th Monitor. Res. Rev., Portsmouth, Virginia.

Street, R. L., Herrmann, R. B., and Nuttli, O. W. (1975), Spectral characteristics of the $L_{g}$ wave generated by central United States earthquakes, Geophys. J. R. Astro. 41, 51-63.

Taylor, S., Velasco, A., Hartse, H., Phillips, W. S., Walter, W. R., and Rodgers, A. (2002), Amplitude corrections for regional discrimination, Pure. App. Geophys. 159, 623-650.

WALD, D. J., and Allen, T. I. (2009), On the use of high-resolution topographic data as a proxy for seismic site conditions $\left(V_{S} 30\right)$, Bull. Seismol. Soc. Am. 99(2A), 935-945, doi:10.1785/0120080255, http://earthquake.usgs.gov/research/hazmaps/interactive/vs30/.

Walter, W. R., Mayeda, K., Malagnini, L., and Scognamiglio, L. (2007), Regional body-wave attenuation using a coda source normalization method: Application to MEDNET records of earthquakes in Italy, Geophys. Res. Lett. 34, L10308.

WALteR, W. R. and TAYLOR, S. R. (2001), A revised magnitude and distance amplitude correction (MDAC2) procedure for regional seismic discriminants, Lawrence Livermore National Laboratory Report UCRL-ID-146882, http://www.llnl.gov/tid/lof/documents/ pdf/240563.pdf.

Walter, W., Mayeda, K., Rodgers, A., Taylor, S., Dodge, D., Matzel, E., and Ganzberger, M. (2004), Regional seismic identification research: processing, transportability and source models, Proc. 26th Seismic Res. Rev. Orlando, Florida.

Xie, J., Wu, A., Liu, R., Schaff, D., Liu, Y., and Liang, J. (2006), Tomographic regionalization of crustal Lg $Q$ in eastern Eurasia, Geophys. Res. Lett. 33, L03315, doi:10.1029/2005GL024410.

Zhang, T.-R., and LAY, T. (1995), Why the Lg phase does not traverse oceanic crust, Bull. Seismol. Soc. Am. 85(6), 16651678 .

(Received October 6, 2008, revised August 10, 2009, accepted September 2, 2009, Published online December 11, 2009) 